\title{
Time Course of Carbendazim Stimulation on Pathogenicity of Sclerotinia sclerotiorum Indicates a Direct Stimulation Mechanism
}

\author{
Ya-Li Di, Xiao-Ming Lu, Zu-Qing Zhu, and Fu-Xing Zhu, College of Plant Science and Technology, Huazhong Agricultural University, \\ Wuhan, 430070, China
}

\begin{abstract}
Di, Y.-L., Lu, X.-M., Zhu, Z.-Q., and Zhu, F.-X. 2016. Time course of carbendazim stimulation on pathogenicity of Sclerotinia sclerotiorum indicates a direct stimulation mechanism. Plant Dis. 100:1454-1459.

Previous studies have demonstrated that subtoxic doses of carbendazim have a stimulatory effect on pathogenicity of Sclerotinia sclerotiorum on rapeseed plants. The present study focused on the time-course profile of the stimulatory effect and its relevance to stimulation mechanisms. At $12 \mathrm{~h}$ postinoculation (HPI), initial necrotic lesions were visible only for rapeseed leaves treated with carbendazim at 0.2 and $1 \mu \mathrm{g} / \mathrm{ml}$, whereas no disease symptoms were observed for the nontreated control. At 18 HPI, carbendazim stimulation on pathogenicity was more obvious than at 12 HPI. Study with scanning electron microscopy demonstrated that no discernable differences in the development of disease symptoms could be detected at 8 HPI. However, at 12 HPI, necrotic symptoms of the epidermal cells were apparent only for leaves sprayed with carbendazim. These results indicated that stimulations on pathogenicity occurred in the first $12 \mathrm{~h}$, implying that direct stimulation rather than overcompensation to the disruption of homeostasis was likely to be the underlying

mechanism for pathogenicity stimulation. Greenhouse experiments showed that spraying carbendazim at $400 \mu \mathrm{g} / \mathrm{ml}$ on potted rapeseed plants had statistically significant $(P<0.05)$ stimulations on pathogenicity for inoculations at 1,3,5, and 7 days after application (DAA). The stimulation action eventually disappeared for inoculations at 14 DAA. Mycelia grown on potato dextrose agar (PDA) supplemented with carbendazim at $400 \mu \mathrm{g} / \mathrm{ml}$ were more pathogenic than the nontreated control. However, after additional growth of the mycelia on fungicide-free PDA for 2 days, the stimulatory effect disappeared completely, indicating that carbendazim was indispensable for pathogenicity stimulations. Studies on biochemical mechanisms indicated that cell-wall-degrading enzymes such as cellulase, pectinase, and polygalacturonase were not involved in pathogenicity stimulations. These results will advance our understanding of the nature and mechanisms of fungicide stimulation on fungal pathogenicity and, thus, are valuable for judicious applications of fungicides.
\end{abstract}

The filamentous ascomycete fungus Sclerotinia sclerotiorum (Lib.) de Bary is a devastating necrotrophic plant pathogen. This pathogen is notorious for its extremely wide host range and can infect more than 400 species of dicotyledonous plants, including many economically important crops (Boland and Hall 1994; Bolton et al. 2006). Diseases caused by $S$. sclerotiorum have been difficult to control because resistant cultivars have not yet been adequately developed and sclerotia are capable of surviving many years in the soil, thus making crop rotations problematic (Adams and Ayres 1979). Application of fungicides is still the mainstay of management measures, and extensive applications have inevitably led to fungicide resistance (Wang et al. 2014). Despite of high levels of carbendazim resistance in eastern China and sporadic occurrences of dimethachlon resistance in major rapeseed-growing regions, carbendazim and dimethachlon remain the two most widely used fungicides for control of $S$. sclerotiorum in China (Wang et al. 2014; Zhang et al. 2003; Zhou et al. $2014 b$ ). However, our previous studies have demonstrated that subtoxic doses of carbendazim and dimethachlon have significant stimulatory effects on pathogenicity of $S$. sclerotiorum on rapeseed plants (Di et al. 2015; Zhou et al. 2014a).

Stimulatory effects of subtoxic doses of fungicides on mycelial growth and pathogenicity of plant pathogens are closely relevant to plant disease management because a small number of pathogen isolates will inevitably be exposed to subtoxic doses of fungicides under field conditions (Flores and Garzón 2013). Stimulatory actions of subtoxic doses of fungicides have been reported in a number of plantpathogenic fungi and oomycetes, such as increased germination of thiabendazole-resistant isolates of Penicillium expansum caused by

Corresponding author: F.-X. Zhu; E-mail: zhufuxing@mail.hzau.edu.cn

Accepted for publication 18 February 2016.

http://dx.doi.org/10.1094/PDIS-11-15-1349-RE

(C) 2016 The American Phytopathological Society low concentrations of thiabendazole (Baraldi et al. 2003); increased mycelial growth of Lyophyllum palustre (Peck) Singer by propamocarb (Landry et al. 2011) and Pythium aphanidermatum, P. irregulare, and P. ultimum by propamocarb (Moorman and Kim 2004); and increased mycelial growth and pathogenicity of metalaxylresistant Phytophthora infestans by metalaxyl (Zhang et al. 1997) and Pythium aphanidermatum by mefenoxam (Garzón et al. 2011). These stimulations of subtoxic doses of fungicides seem to be a general phenomenon commonly called hormesis, and chemical hormesis has been reported across a tremendously broad range of biological systems and a wide variety of chemical agents (Calabrese 2013; Calabrese and Blain 2011).

Hormetic mechanisms involving cellular receptors and cell signaling pathways have been extensively documented and reviewed (Calabrese 2013; Calabrese and Blain 2011). The possible mechanisms for hormesis include direct stimulation, overcompensation to a disruption of homeostasis, and superimposition of two monotonic dose responses, with one taking effect at lower doses and the other taking effect at higher doses (Calabrese 2001). It is highly likely that multiple metabolic processes are involved in hormetic responses, some acting during the stimulation phase and others acting during the inhibition phase (Calabrese 2008; Garzón and Flores 2013). Up to the present, little has been known about mechanisms of fungicide hormesis. In the case of carbendazim stimulations on virulence of S. sclerotiorum, our previous studies have indicated that pathogenicity determinant oxalic acid and sensitivity to reactive oxygen species were not involved, whereas dimethachlon stimulations on pathogenicity were correlated with higher tolerances to oxidative stress that would confer an advantage to $S$. sclerotiorum in infecting host plants (Di et al. 2015; Heller and Tudzynski 2011; Zhou et al. 2014a). A time-course profile of stimulations may give hints about the underlying hormetic mechanisms. Up to now, there has been no detailed report on the time course of fungicide hormesis, perhaps mainly due to difficulties in attaining reproducible and reliable experimental results, especially when very low concentrations of fungicides are used, as is often the case for hormesis studies. The objects of the 
present study were to (i) determine the time course of carbendazim stimulations on pathogenicity of S. sclerotiorum and (ii) explore potential mechanisms for carbendazim hormesis.

\section{Materials and Methods}

Isolates of $\mathbf{S}$. sclerotiorum. Two field isolates (LJ-86 and AH-17) of $S$. sclerotiorum were collected from diseased rapeseed plants with typical symptoms of Sclerotinia stem rot in Anhui Province of China in 2012. Mature sclerotia were stored at $4^{\circ} \mathrm{C}$ in a refrigerator before being cultured on potato dextrose agar (PDA) media. The $50 \%$ effective concentration $\left(\mathrm{EC}_{50}\right)$ values for carbendazim to the two isolates were higher than $1,000 \mu \mathrm{g} / \mathrm{ml}$ (Di et al. 2015).

Fungicide. Technical-grade carbendazim $(98.1 \%$ active ingredient; Tian Jin Jin Bei Chemical Co. Ltd.) was dissolved in hydrochloric acid $(\mathrm{HCl}, 0.1 \mathrm{~mol} / \mathrm{liter}$ ) to produce a stock solution of $8,000 \mu \mathrm{g} / \mathrm{ml}$. The stock solution was stored at $4^{\circ} \mathrm{C}$ in a refrigerator for no longer than 2 weeks before being serially diluted for experiments.

Time course of pathogenicity stimulation of subtoxic doses of carbendazim on $S$. sclerotiorum on detached leaves of rapeseed plants. Determination of pathogenicity stimulation of carbendazim on detached leaves of rapeseed plants was performed according to Di et al. (2015), with slight modifications. Briefly, leaves of 8 to $11 \mathrm{~cm}$ in diameter were detached from 4- to 5-week-old rapeseed plants growing in the greenhouse at about $25^{\circ} \mathrm{C}$. The detached leaves were rinsed with sterile water, air dried on a clean bench, and transferred to 15 -cm-diameter petri dishes lined with wetted filter paper to maintain high humidity. Carbendazim solutions were prepared by diluting carbendazim stock solution with sterilized $0.1 \%$ surfactant Triton-100 in water to final concentrations of 0.2 and $1 \mu \mathrm{g} / \mathrm{ml}$. $\mathrm{HCl}$ at the same final concentration as in the diluted carbendazim solutions was used as the nontreated control. Detached leaves were sprayed with carbendazim or $\mathrm{HCl}$ dilutions by a handheld sprayer ( $800 \mathrm{ml}$ in volume; Xinmeir Co. Ltd.). The treated leaves were air dried for $1 \mathrm{~h}$, then inoculated on the adaxial surface with inverted 5-mm-diameter mycelial plugs (one plug per leaf). The inoculated leaves in petri dishes were incubated at $25^{\circ} \mathrm{C}$ in a growth chamber, with relative humidity maintained over $85 \%$. Disease symptoms were recorded and photographed with a digital camera at $8,12,18,36$, and $48 \mathrm{~h}$ postinoculation (HPI). The experiment was performed in triplicate and repeated independently three times.

Microscopic observations of infection process with scanning electron microscopy. Detached leaves inoculated with mycelial plugs were sampled at 4, 8, and 12 HPI. Observations with scanning electron microscopy (SEM) were according to Zhang et al. (2010), with minor modifications. Briefly, the inoculated segments of leaves were cut into small pieces ( 3 by $3 \mathrm{~mm}$ ). The samples were fixed in $2.5 \%(\mathrm{wt} / \mathrm{vol})$ glutaraldehyde solution in sodium phosphate buffer $(0.05 \mathrm{M}, \mathrm{pH} 7.0)$ at $4^{\circ} \mathrm{C}$ for $24 \mathrm{~h}$, washed three times in $0.05 \mathrm{M}$ sodium phosphate buffer for $10 \mathrm{~min}$ each time, then dehydrated in a graded ethanol series. After critical point drying and gold-coating in a sputter coater (JFC-1600; NTC, Japan), the leaf specimens were examined for infection process with SEM (JSM-6390/LV; NTC, Japan).

Pathogenicity stimulation duration for inoculations on potted rapeseed plants. Determination of pathogenicity stimulation duration of spraying carbendazim on potted rapeseed plants was conducted with a similar procedure as described above. Potted rapeseed plants (about 4 weeks old) grown in 20-cm-diameter pots containing a composite mixture of peat and soil (weight ratio $=1: 1$ ) were sprayed with carbendazim at $400 \mu \mathrm{g} / \mathrm{ml}$ until runoff, with rapeseed plants treated

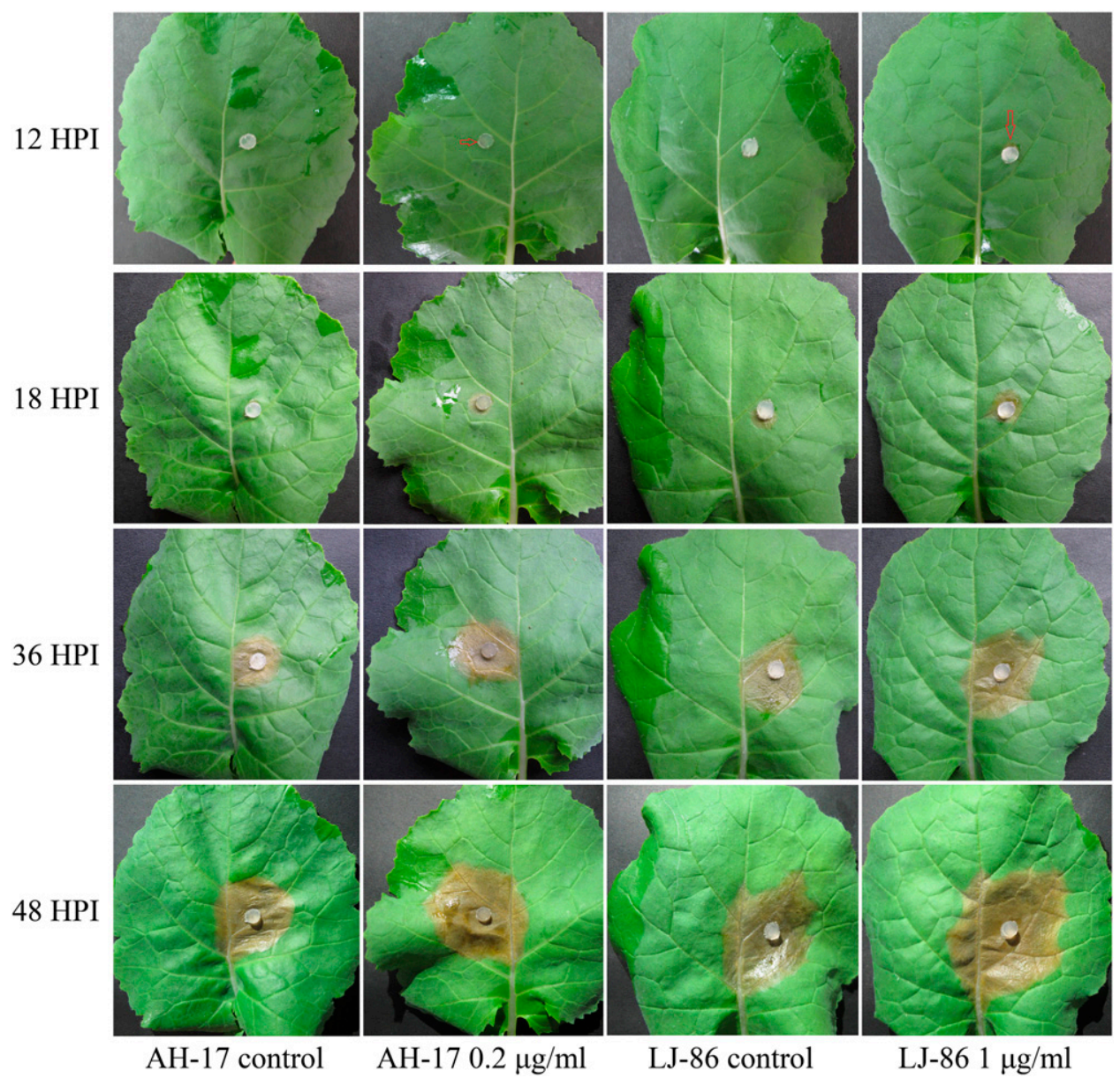

Fig. 1. Time course of infection process of Sclerotinia sclerotiorum isolates $\mathrm{AH}-17$ and $\mathrm{LJ}-86$ on leaves of rapeseed plants. Leaves treated with $0.1 \%$ Triton-100 in water were used as the nontreated control (the first and third columns). Concentrations of carbendazim for isolate AH-17 (the second column) and for LJ-86 (the last column) were 0.2 and $1 \mu \mathrm{g} / \mathrm{ml}$, respectively. Arrows indicate first sign of infection at $12 \mathrm{~h}$ postinoculation $(\mathrm{HPI})$ for leaves treated with carbendazim. 
with $\mathrm{HCl}$ at $0.004 \mathrm{~mol} / \mathrm{liter}$ in $0.1 \%$ Triton- 100 as the nontreated control. The treated rapeseed plants were incubated as described above. In all, $0,1,3,5,7,10$, and 14 days after application (DAA), each of the three plants treated with carbendazim at $400 \mu \mathrm{g} / \mathrm{ml}$ and three plants treated with $\mathrm{HCl}$ as the control were inoculated on the adaxial surface of three leaves (with one mycelial plug per leaf). The inoculated plants were incubated as described above. Lesion diameters were measured twice at right angles after $48 \mathrm{~h}$ of incubation. The experiment was repeated independently six times.

Stimulatory effects of carbendazim-amended PDA on pathogenicity of $S$. sclerotiorum. Determination of the stimulatory effects of growth on PDA supplemented with carbendazim on pathogenicity was conducted according to Zhou et al. (2014a). Leaves of 8 to $11 \mathrm{~cm}$ in diameter were detached from 4- to 5-week-old oilseed rape plants grown in the greenhouse at $25^{\circ} \mathrm{C}$. The detached leaves were rinsed with sterile water, air dried on a clean bench, and transferred to $15-\mathrm{cm}-$ diameter petri dishes with water-soaked sterilized filter paper to maintain high humidity. Inverted mycelial plugs ( $5 \mathrm{~mm}$ in diameter) cut from fresh margins of 2-day-old colonies grown on PDA amended with carbendazim at a final concentration of $400 \mu \mathrm{g} / \mathrm{ml}$ were inoculated on the adaxial surface of the detached leaves. Leaves inoculated with mycelial plugs from colonies grown on fungicide-free PDA amended with $\mathrm{HCl}$ were used as the nontreated control. The inoculated leaves were incubated as described above. Lesion diameters were measured twice at right angles after $48 \mathrm{~h}$ of incubation. In order to study the lasting stimulatory effects of carbendazim, mycelial plugs from PDA supplemented with carbendazim at $400 \mu \mathrm{g} / \mathrm{ml}$ were transferred to PDA free of fungicide and incubated for an additional 2 days; then, mycelial plugs from the PDA without carbendazim were inoculated to detached leaves of rapeseed plants as described above. Lesion diameters were measured as described above. The experiment was conducted in triplicate and repeated independently three times.

Effect of subtoxic doses of carbendazim on activities of cellwall-degrading enzymes. Mycelia were grown on cellophaneoverlaid PDA amended with carbendazim at 1 and $5 \mu \mathrm{g} / \mathrm{ml}$ for 3 days. PDA supplemented with $\mathrm{HCl}$ at $0.001 \mathrm{~mol} / \mathrm{liter}$ was used as the nontreated control. Mycelia were harvested by gentle scraping with a scoop. About $0.5 \mathrm{~g}$ of fresh mycelia was weighed, lyophilized, and ground into fine powder with a mortar. Cold sodium acetate-acetic acid buffer $\left(4^{\circ} \mathrm{C}, 1.5 \mathrm{ml}, 50 \mathrm{mmol} / \mathrm{liter}, \mathrm{pH} 5.0\right)$ was added to suspend the powder. After centrifugation at $10,000 \times g$ and $4^{\circ} \mathrm{C}$ for $10 \mathrm{~min}$,
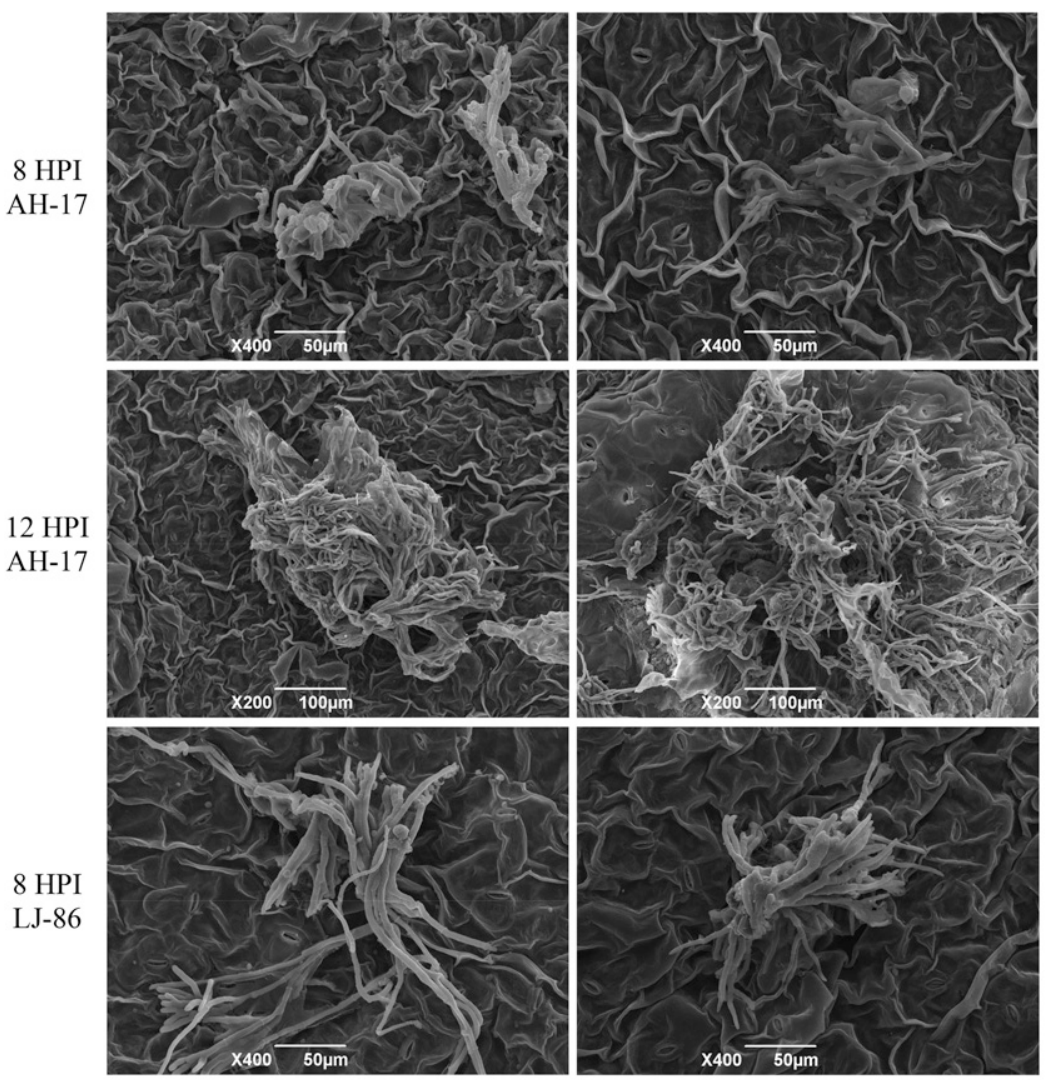

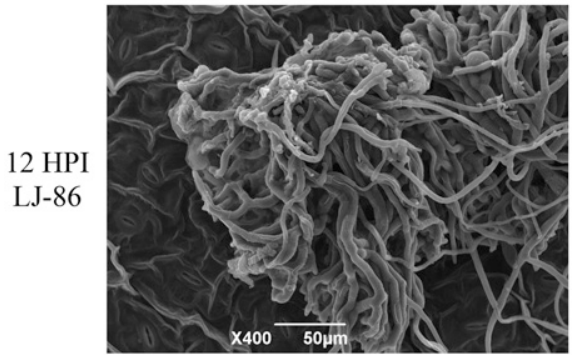

Untreated control

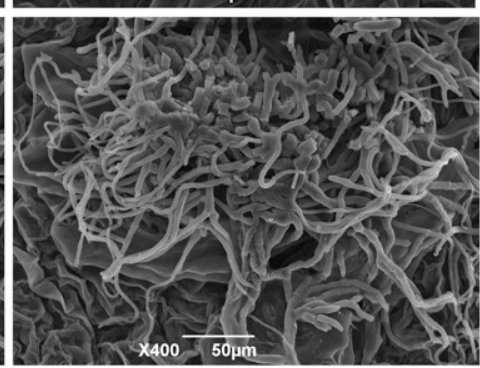

carbendazim at $1 \mu \mathrm{g} / \mathrm{ml}$

Fig. 2. Scanning electron micrographs showing infection processes of Sclerotinia sclerotiorum isolates AH-17 and LJ-86 on rapeseed leaves sprayed with $0.1 \%$ Triton- 100 in water as the nontreated control (the first column) and sprayed with carbendazim at $1 \mu \mathrm{g} / \mathrm{ml}$. At $12 \mathrm{~h}$ postinoculation (HPI), disease symptoms of necrotic epidermis were obvious for leaves treated with carbendazim. 
the supernatant was used to assay activities of pectinase and polygalacturonase. For determination of cellulase activity, citric acid at $50 \mathrm{mmol} /$ liter-sodium citrate buffer $(\mathrm{pH} \mathrm{4.4)}$ instead of sodium acetate-acetic acid buffer was used to suspend the powder.

Cellulase activity was measured spectrophotometrically using dinitrosalicylic acid (DNS) as the dyeing agent, according to Bai and Wang (2012). DNS (3 g; Shanghai Chemical Reagent Co. Ltd.) was dissolved in $500 \mathrm{ml}$ of distilled water at $45^{\circ} \mathrm{C}$ in a 1,000 - $\mathrm{ml}$ brown volumetric flask. The following reagents were added sequentially into the volumetric flask: $10.4 \mathrm{~g}$ of $\mathrm{NaOH}, 90.1 \mathrm{~g}$ of potassium sodium tartrate, $2.5 \mathrm{~g}$ of phenol, and $2.5 \mathrm{~g}$ of anhydrous sodium sulfite. Distilled water was added to the final volume of $1,000 \mathrm{ml}$. Sodium carboxymethyl cellulose (CMC; $0.5 \%)$ dissolved in citric acid-sodium citrate buffer at $50 \mathrm{mmol} /$ liter was used as the substrate. The activity of cellulase was determined by the conversion of CMC to glucose. The reaction mixtures initially contained $1 \mathrm{ml}$ of supernatant enzyme and $3 \mathrm{ml}$ of $0.5 \% \mathrm{CMC}$ and were maintained at $50^{\circ} \mathrm{C}$ for $30 \mathrm{~min}$. The

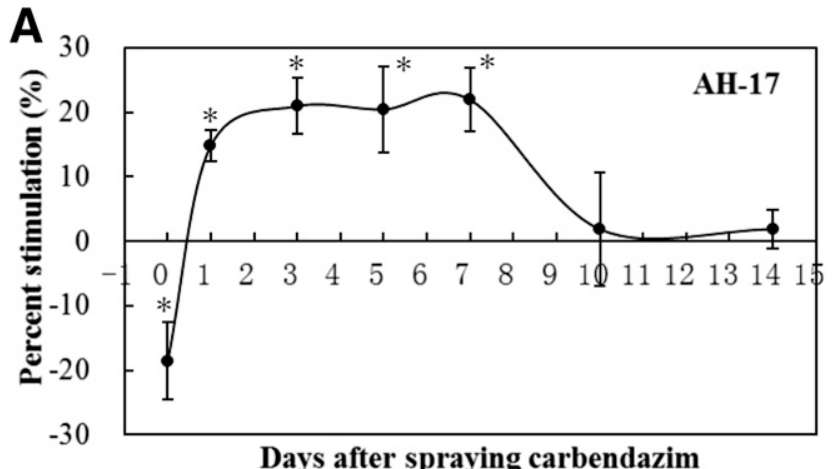

B

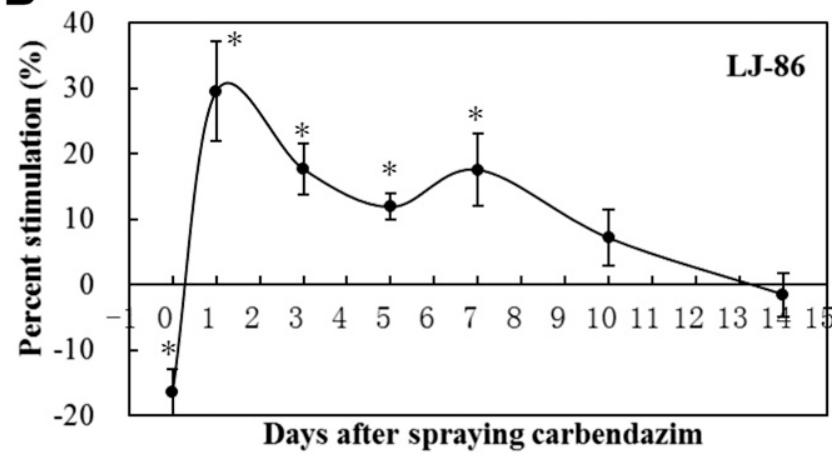

Fig. 3. Pathogenicity stimulation for inoculations of two Sclerotinia sclerotiorum isolates, A, AH-17 and B, LJ-86, at different time points after applying carbendazim at $400 \mu \mathrm{g} / \mathrm{ml}$ on potted rapeseed plants. Error bars denote standard error of the mean for six replicates. Asterisks denote significant $(\alpha=0.05)$ difference from the control. reaction was stopped by heating in boiling water for $10 \mathrm{~min}$; then, $3 \mathrm{ml}$ of DNS solution was added for the color reaction. Absorbance was measured at $530 \mathrm{~nm}$ with a UV-1800 spectrophotometer (Shimadzu Corporation). Reaction mixture with heat-inactivated enzyme was used as the control. A standard curve was developed using a series of glucose solutions. Reaction unit was defined as the production of glucose at $1 \mathrm{mg} / \mathrm{min}$ at $50^{\circ} \mathrm{C}$.

Pectinase activity was assayed by the method of DNS according to Moyo et al. (2003). The reaction mixture was composed of $2 \mathrm{ml}$ of $0.5 \%$ pectin in acetate buffer at $0.05 \mathrm{~mol} / \mathrm{liter}, 7.5 \mathrm{ml}$ of acetate buffer ( $\mathrm{pH} 5.0$ ), and $0.5 \mathrm{ml}$ of supernatant enzyme. The mixture was incubated at $45^{\circ} \mathrm{C}$ for $30 \mathrm{~min}$ in a water bath incubator shaker. Reaction mixture $(1 \mathrm{ml})$ was pipetted to a centrifuge tube and $1 \mathrm{ml}$ each of DNS and $40 \%$ potassium sodium tartrate were added. After being vortexed and maintained in boiling water for $10 \mathrm{~min}$, absorbance was measured at $575 \mathrm{~nm}$ with a UV-1800 spectrophotometer. One unit of pectinase activity was defined as the amount of the enzyme that catalyzed the formation of galacturonic acid at $1 \mu \mathrm{mol} / \mathrm{min}$ at $45^{\circ} \mathrm{C}$. Polygalacturonase activity was determined by a similar procedure, with $0.5 \%$ galacturonic acid instead of pectin as the substrate. The experiment was performed in triplicate and repeated independently three times.

Data analysis. Percentage stimulations were calculated by the following formula, with the diameter of mycelial plugs being subtracted from the measured lesion diameters: percentage stimulation $(\%)=$ [(lesion diameter of the treated - lesion diameter of the control)/lesion diameter of the control] $\times 100 \%$.

Percentage stimulations were arcsine square-root transformed before statistical analysis. One-way analysis of variance and Dunnett's test $(P=0.05)$ in SPSS (Statistical Product and Service Solutions, ver. 19.0; SPSS Inc.) were employed to evaluate whether the percentage stimulations were significantly higher than the control. In pot experiments, the experimental unit was defined as each rapeseed plant grown in one pot, with the arithmetic mean of lesion diameters as the response. Figures were created with GraphPad Prism (ver. 5.01; GraphPad Software, Inc.) and Microsoft Excel (ver. 2010; Microsoft Corporation).

\section{Results}

Pathogenicity stimulations on detached leaves. The study on infection process of detached leaves demonstrated that, at $8 \mathrm{HPI}$, no necrotic lesions could be observed for all the treatments. At 12 HPI, initial necrotic lesions were visible only for leaves treated with subtoxic doses of carbendazim, whereas no necrotic lesions were detected for the nontreated control (Fig. 1). From 18 to 48 HPI, pathogenicity stimulations of carbendazim at 0.2 and $1 \mu \mathrm{g} / \mathrm{ml}$ were obvious for both isolates compared with the control.

Infection process shown by SEM. Observations with SEM gave similar results and, at $4 \mathrm{HPI}$, no infection structure such as infection cushions could be detected on leaves. At 8 HPI, infection cushions were visible under SEM but no obvious differences could be detected between the nontreated control and those treated with carbendazim
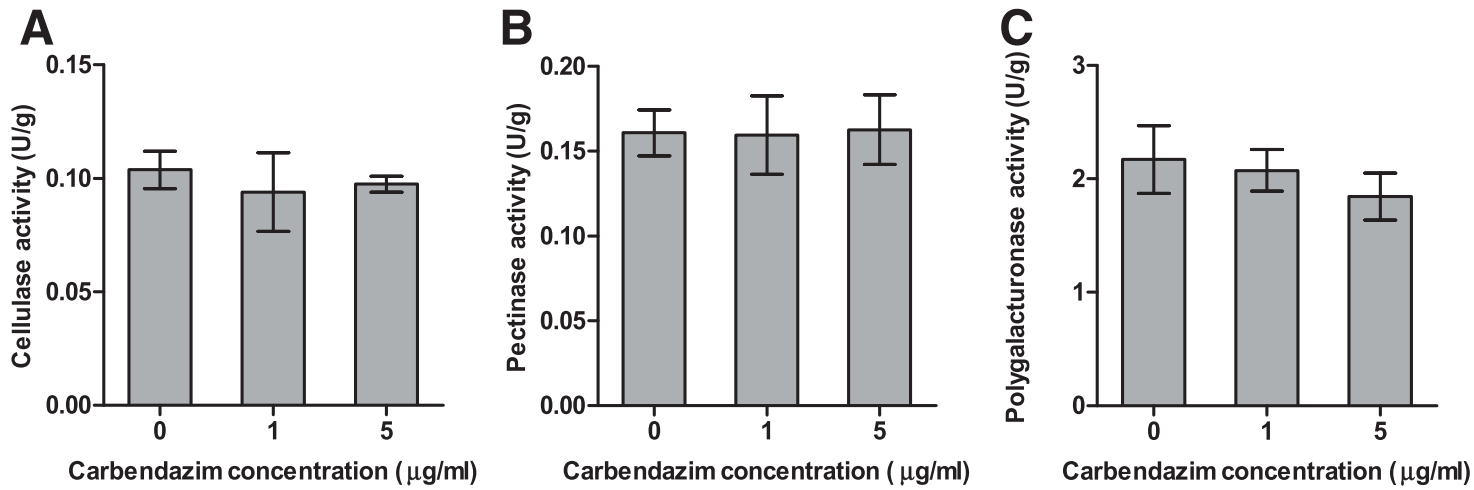

Fig. 4. Effects of carbendazim on activities of cell-wall-degrading enzymes A, cellulase; B, pectinase; and C, polygalacturonase in Sclerotinia sclerotiorum isolate AH-17. Error bars denote standard deviation of three replicates. 
(Fig. 2). At 12 HPI, necrotic symptoms of the epidermal cells were more apparent for leaves treated with subtoxic doses of carbendazim than for the nontreated control.

Pathogenicity stimulation duration for inoculations on potted rapeseed plants. For the two carbendazim-resistant isolates, carbendazim at $400 \mu \mathrm{g} / \mathrm{ml}$ had an inhibitory effect only for inoculations immediately after its application. Significant $(P<0.05)$ stimulations on pathogenicity were detected for inoculations from 1 to 7 DAA (Fig. 3). Stimulation magnitude decreased for inoculations at 10 DAA. For inoculations at 14 DAA, pathogenicity stimulations disappeared completely.

Effects of carbendazim-amended PDA on pathogenicity. With a percent inhibition of $-1.82 \%$, mycelial growth rate of isolate AH-17 on PDA supplemented with carbendazim at $400 \mu \mathrm{g} / \mathrm{ml}$ was similar to that of the nontreated control. Inoculations to detached leaves with mycelial plugs from carbendazim-amended PDA displayed a pathogenicity stimulation of $19.7 \%$. However, after transferring the mycelia onto PDA without carbendazim and growing for additional 2 days, the stimulatory effect on pathogenicity disappeared completely.

Effect of subtoxic doses of carbendazim on activities of cellwall-degrading enzymes. Compared with the nontreated control, mycelia grown on PDA supplemented with carbendazim at 1 and $5 \mu \mathrm{g} / \mathrm{ml}$ did not have increased but, instead, slightly decreased activities for the three cell-wall-degrading enzymes (Fig. 4). Statistically, carbendazim at 1 and $5 \mu \mathrm{g} / \mathrm{ml}$ had no significant effects on activities of cellulase $(P=0.332)$, pectinase $(P=0.961)$, and polygalacturonase $(P=0.142)$.

\section{Discussion}

The present study showed that the earliest evidence of carbendazim stimulation on S. sclerotiorum pathogenicity could be detected with SEM at $12 \mathrm{HPI}$ and with the naked eye at $18 \mathrm{HPI}$. At $12 \mathrm{HPI}$, the necrotic lesions on the epidermis of rapeseed leaves were more obvious on leaves treated with carbendazim than the nontreated control. At $8 \mathrm{HPI}$, infection cushions could be observed with SEM on inoculated leaves of rapeseed but no apparent differences could be detected between the nontreated control and the leaves sprayed with carbendazim at $1 \mu \mathrm{g} / \mathrm{ml}$. At $4 \mathrm{HPI}$, no infection cushions were detected with SEM in the present study. Studies by Xiao et al. (2014) demonstrated that infection cushions of $S$. sclerotiorum could be observed with SEM on intact Arabidopsis thaliana leaves at 4 HPI. Host plant species and humidity may influence the infection progress. The fact that stimulation on lesion development on the epidermis of rapeseed leaves could be observed as early as 12 HPI indicated that a direct stimulation mechanism rather than overcompensation to disruption of homeostasis may account for the pathogenicity stimulations of carbendazim on S. sclerotiorum. Nevertheless, overcompensation to disruption of homeostasis has been inferred as a potential hormetic mechanism from the phenomena of early inhibition and subsequent stimulation. For example, it has been reported that plant growth could be inhibited by a chemical stressor at $24 \mathrm{~h}$ but would be stimulated at $72 \mathrm{~h}$ (Calabrese 2015b; Townsend 1897).

The direct stimulation mechanism for chemical hormesis has been reported for growth stimulation of herbicide phosphinothricin on the flowering plant Lotus corniculatus L. Growth stimulation at lower phosphinothricin concentrations was caused by activation of chloroplastic isoform GS2, while growth inhibition at higher phosphinothricin concentrations was a result of inhibition of chloroplastic isoform GS1 and GS2 (Dragićević et al. 2013). The direct stimulation mechanism may also account for many low-dose stimulatory responses in pharmacology literature (Calabrese 2015a). Studies with pharmacological receptor models demonstrated that the direct stimulations by low doses of chemicals may be caused by two distinct receptor subtypes. One subtype with stimulatory effects has fewer receptors but greater affinity for the agonist, whereas the other subtype with inhibitory effects has more receptors but lower affinity for the agonist. Superimposition of the effects of the two receptor subtypes will lead to stimulation at lower doses and inhibition at higher doses (Calabrese 2013, 2015b).
It should be noted that hormetic dose responses are independent of biological models, endpoints measured, chemical classes, and mechanisms involved. Hormesis (i.e., low-dose stimulation and high-dose inhibition or vice versa) is a common and broadly generalizable phenomenon, and may represent phenotypic plasticity and adaption of an organism to environment (Calabrese and Mattson 2011). Although quantitative features of hormesis are independent of hormetic mechanisms, studies on time-course profile of hormetic dose response can give hints about the underlying mechanisms for hormesis. Of course, studies on biochemical and molecular mechanisms of hormesis are needed to advance our understanding of the nature of hormesis and, in the case of carbendazim stimulation on S. sclerotiorum pathogenicity, insight into the stimulatory mechanisms will be conducive to judicious application of this fungicide for control of S. sclerotiorum.

Carbendazim is commonly applied at around $400 \mu \mathrm{g} / \mathrm{ml}$ on rapeseed plants to control Sclerotinia stem rot in China and can provide excellent control on carbendazim-sensitive isolates of S. sclerotiorum. However, the present study demonstrated that, for carbendazimresistant isolates, carbendazim at $400 \mu \mathrm{g} / \mathrm{ml}$ had significant pathogenicity stimulations for inoculations 1 to 7 DAA (Fig. 3). Given the development and prevalence of carbendazim resistance in plant pathogens, especially in S. sclerotiorum in eastern China (Wang et al. 2014; Zhu et al. 2016), this has significant implications for applications of carbendazim. This may also explain why Sclerotinia stem rot might become aggravated after application of carbendazim, as occasionally noticed by rapeseed growers (Di et al. 2015).

The first barrier to fungal infection is the plant cell wall, which is mainly composed of cellulose, xyloglucan, pectin, and protein. Plant pathogens release a battery of degrading enzymes, most notably cellulase, pectinase, and polygalacturonase, to weaken the plant cell wall and facilitate their infections (Bashi et al. 2012). However, the present study demonstrated that pathogenicity stimulation by carbendazim was not caused by any of the three cell-wall-degrading enzymes. Biochemical and molecular mechanisms for carbendazim stimulation remain to be elucidated in the future.

\section{Acknowledgments}

This study was supported by National Natural Science Foundation of China (31371964) and the Special Fund for Agro-scientific Research in the Public Interest (201103016).

\section{Literature Cited}

Adams, P. B., and Ayres, B. E. 1979. Ecology of Sclerotinia species. Phytopathology 69:896-899.

Bai, Y., and Wang, W. X. 2012. Activity measuring methods for proteinase, amylase, lipase and cellulase in intestine of Apostichopus japonicus (Selenka). Feed Ind. 33:28-32.

Baraldi, E., Mari, M., Chierici, E., Pondrelli, M., Bertolini, P., and Pratella, G. C. 2003. Studies on thiabendazole resistance of Penicillium expansum of pears: Pathogenic fitness and genetic characterization. Plant Pathol. 52:362-370.

Bashi, Z. D., Rimmer, S. R., Khachatourians, G. G., and Hegedus, D. D. 2012 Factors governing the regulation of Sclerotinia sclerotiorum cutinase A and polygalacturonase 1 during different stages of infection. Can. J. Microbiol. 58:605-616.

Boland, G. J., and Hall, R. 1994. Index of plant hosts of Sclerotinia sclerotiorum Can. J. Plant Pathol. 16:93-108.

Bolton, M. C., Thomma, B. P., and Nelson, B. D. 2006. Sclerotinia sclerotiorum (Lib.) de Bary: Biology and molecular traits of a cosmopolitan pathogen. Mol. Plant Pathol. 7:1-16.

Calabrese, E. J. 2001. Overcompensation stimulation: A mechanism for hormetic effects. Crit. Rev. Toxicol. 31:425-470.

Calabrese, E. J. 2008. Hormesis and medicine. Br. J. Clin. Pharmacol. 66:594-617.

Calabrese, E. J. 2013. Hormetic mechanisms. Crit. Rev. Toxicol. 43:580-606.

Calabrese, E. J. 2015a. Hormesis: Principles and applications. Homeopathy 104: 69-82.

Calabrese, E. J. 2015b. Hormesis within a mechanistic context. Homeopathy 104 90-96.

Calabrese, E. J., and Blain, R. B. 2011. The hormesis database: The occurrence of hormetic dose-responses in the toxicological literature. Regul. Toxicol. Pharmacol. 61:73-81.

Calabrese, E. J., and Mattson, M. P. 2011. Hormesis provides a generalized quantitative estimate of biological plasticity. J. Cell Commun. Signal. 5:25-38.

Di, Y. L., Zhu, Z. Q., Lu, X. M., and Zhu, F. X. 2015. Pathogenicity stimulation of Sclerotinia sclerotiorum by subtoxic doses of carbendazim. Plant Dis. 99:13421346. 
Dragićević, M., Platiša, J., Nikolić, R., Todorović, S., Bogdanović, M., Mitić, N., and Simonović, A. 2013. Herbicide phosphinothricin causes direct stimulation hormesis. Dose Response 11:344-360.

Flores, F. J., and Garzón, C. D. 2013. Detection and assessment of chemical hormesis on the radial growth in vitro of oomycetes and fungal plant pathogens. Dose Response 11:361-373.

Garzón, C. D., and Flores, F. J. 2013. Hormesis: Biphasic dose-response to fungicide in plant pathogens and their potential threat to agriculture. In: Fungicides-Showcases of Integrated Plant Disease Management from Around the World. M. Nita, ed. Online publication. Intech. doi:10.5772/55359

Garzón, C. D., Molineros, J. E., Yanez, J. M., Flores, F. J., Jimenez-Gasco, M. M., and Moorman, G. W. 2011. Sublethal doses of mefenoxam enhance Pythium damping-off of geranium. Plant Dis. 95:1233-1238.

Heller, J., and Tudzynski, P. 2011. Reactive oxygen species in phytopathogenic fungi: Signaling, development, and disease. Annu. Rev. Phytopathol. 49:369390.

Landry, J., Martinez, C., and Rochefort, L. 2011. The use of fungicide Nova to mitigate infection of sphagnum by parasitic fungi in the greenhouse. Botany 89:655-661.

Moorman, G. W., and Kim, S. H. 2004. Species of Pythium from greenhouses in Pennsylvania exhibit resistance to propamocarb and mefenoxam. Plant Dis. 88:630-632.

Moyo, S., Gashe, B. A., Collison, E. K., and Mpuchane, S. 2003. Optimising growth conditions for the pectinolytic activity of Kluyveromyces wickerhamii by using response surface methodology. Int. J. Food Microbiol. 85:87-100.
Townsend, C. O. 1897. The correlation of growth under the influence of injuries Ann. Bot. (Lond.) 11:509-532.

Wang, Y., Hou, Y. P., Chen, C. J., and Zhou, M. G. 2014. Detection of resistance in Sclerotinia sclerotiorum to carbendazim and dimethachlon in Jiangsu Province of China. Australas. Plant Pathol. 43:307-312.

Xiao, X., Xie, J., Cheng, J., Li, G., Yi, X., Jiang, D., and Fu, Y. 2014. Novel secretory protein Ss-Caf1 of the plant-pathogenic fungus Sclerotinia sclerotiorum is required for host penetration and normal sclerotial development. Mol. Plant-Microbe Interact. 27:40-55.

Zhang, L., Wu, M., Li, G., Jiang, D., and Huang, H. 2010. Effect of mitovirus infection on formation of infection cushions and virulence of Botrytis cinerea. Physiol. Mol. Plant Pathol. 75:71-80.

Zhang, S., Panaccione, S. G., and Gallegly, M. E. 1997. Metalaxyl stimulation of growth of isolates of Phytophthora infestans. Mycologia 89:289-292.

Zhang, X. L., Sun, X. M., Zhang, G. F., Yi, H. J., and Qiang, Y. 2003. Preliminary report on the monitoring of the resistance of Sclerotinia sclerotiorum to carbendazim and its integrated management. Chin. J. Pestic. Sci. Adm. 24:18-22.

Zhou, F., Liang, H. J., Di, Y. L., You, H., and Zhu, F. X. 2014a. Stimulatory effects of sublethal doses of dimethachlon on Sclerotinia sclerotiorum. Plant Dis. 98:1364-1370.

Zhou, F., Zhang, X. L., Li, J. L., and Zhu, F. X. 2014b. Dimethachlon resistance in Sclerotinia sclerotiorum in China. Plant Dis. 98:1221-1226.

Zhu, Z. Q., Zhou, F., Li, J. L., Zhu, F. X., and Ma, H. J. 2016. Carbendazim resistance in field isolates of Sclerotinia sclerotiorum in China and its management. Crop Prot. 81:115-121. 